\title{
Business model dynamics: a case survey
}

\author{
Mark de Reuver ${ }^{1}$, Harry Bouwman ${ }^{2}$ and Ian Maclnnes ${ }^{3}$ \\ ${ }^{1}$ Delft University of Technology, g.a.dereuver@tudelft.nl, ${ }^{2}$ Delft University of Technology, \\ w.a.g.a.bouwman@tudelft.nl, ${ }^{3}$ Syracuse University, imacinne@syr.edu \\ Received 17 February 2008; received in revised form 17 May 2008; accepted 30 September 2008
}

\begin{abstract}
In the turbulent world of e-commerce, companies can only survive by continuously reinventing their business models. However, because most studies look at business models as snapshots in time, there is little insight into how changing market-related, technological and regulatory conditions generally drive revisions in business models. In this paper, we examine which types of external drivers are strongest in forcing business models to change throughout their life cycle. To do so, we study 45 longitudinal case descriptions on business model dynamics of (networks of) organizations in various industries. The results of this survey indicate that technological and market-related forces are the most important drivers of business model dynamics, while regulation plays only a minor role. In particular for start-ups, the effect of technological and market-related drivers is the strongest in the early stages of a new business model, while the effects are moderate over time for established, large companies. Our results provide clues to practitioners on what external factors to take into account in different stages of business model design and redesign.
\end{abstract}

Key words: Business models, Business model dynamics, Business model design, Start-ups, Case survey 


\section{Introduction}

To keep up with changing technology, as well as market-related and regulatory conditions, organizations have to revise their business models over time [13]. Design choices that appear to be solid when an initial product or service concept is developed often have to be adapted when the product or service is launched and exploited commercially. Although these changes are to a certain extent endogenous in nature, they are to a significant degree caused by external drivers.

While the need for business model reinvention is well-known [6], there is a considerable focus on studying business models at a certain moment in time. Although recent research has provided some clues about business model dynamics [2], [11], [22], the exact relationship between external forces and the business model design choices firms make remains an unexplored area. Insight into how external events affect business model dynamics would help practitioners keep their business models adaptable and flexible over time. In addition, it would help refining and focusing methodologies for business model (re)design (e.g., [3], [16]). In this paper, we examine what types of external drivers are most important during the subsequent phases of business model life cycles. We look at the business models of (networked) organizations to see how they change over time as a consequence of external drivers, focusing more on the drivers than on the business models as such. To do so, we conduct a case survey [10], [23] involving a large set of existing case descriptions.

In section 2, we provide a brief overview of business model and phasing model literature, and present our research model in section 3. In section 4, we discuss our methodology, and in section 5 we report our results. We close by addressing the limitations of our study in section 6 , and presenting our conclusions in section 7.

\section{Literature Review}

\subsection{Business models}

The concept of business models has its origins in various fields, including computer science, e-business, strategy, supply chain management and information systems [8], [20], and was developed mainly as a response to the need to explicate the value of ICT-driven innovations for organizations. Studying business models serves various purposes, such as understanding the elements and their relationships within specific business domains, communicating and sharing this understanding with the outside world, using the business models as a basis for change, measuring the performance of organizations, simulating and learning about e-business, experimenting with and assessing new business models and changing and improving the current way of doing business [14] - [15]. Since its conception, the focus has shifted from defining the concept, via exploring business model components and developing taxonomies of typical business models, towards developing descriptive models [15]. According to Andries et al. [2], however, no single authoritative definition of what a business model is has yet been provided. We follow the definition of Chesbrough and Roosenbloom, who see a business model as a blueprint for the way a business creates and captures value from new services or products [8]. As such, a business model describes how a company or network of companies aims to make money and create consumer value [11].

In our view, business models are an abstraction of how organizations create value [19]. However, external factors, including socio-economic trends, technological developments and political and legal changes, are important in understanding how business models are used in practice. With a few exceptions [2], [11], [22], most studies adopt a static approach to business models, assuming implicitly that they remain stable over time. However, organizations often need to keep reinventing their business model to stay in touch with fast-changing environments in some sectors [1]. As a result, business models have to be reconsidered during all phases, from business model development to exploitation. Business model dynamics are present in all the components of the business model: the service and/or product being offered, the technical configuration, and the organizational and financial arrangements. In this paper we focus exclusively on the environmental dynamics in relation to the business model. We will not discuss the internal dynamics of a business model [3].

\subsection{Phasing models}

Phasing models help us understand how the environmental dynamics, e.g. innovation and change, affect firm strategies and business models [1], and have appeared in various fields. With regard to the diffusion of innovations, Rogers [18] identifies the phases of problem recognition, basic or applied research, development, commercialization, and finally diffusion and adoption. It makes sense to may assume that the conceptualization of new business models develops in parallel with the process between solving a problem and ensuring market adoption of the innovative solution. According to innovation management literature [21], new products and services follow a life cycle from innovation to development and maturity. Next, they enter a phase where new generations of products and services overtake the by now mature innovation. Rapid and frequent product and service innovation is prevalent in the early phases, while later stages are characterized by stable product and service concepts, with only incremental changes motivated by cost reduction and cost-effective exploitation. In literature on venturing, the development process from 
business idea to established business is divided into different stages. Burgelman [5] describes four processes, which may overlap and be iterative in nature: defining the activities in the innovation project, gaining support within the organization, managing the structural implementation of the new product, and embedding the innovation in the company strategy. Mason and Rohner [12] distinguish the phases of venture vision and validation, alpha offering, beta offering, and market offering. Because different actors, resources and capabilities are needed in each of these phases, the components of the business models are subject to change.

Based on the phasing models we discussed above, we propose dividing the life cycle of business models into three phases: (I) Development/R\&D; (II) Implementation/Roll-out; (III) Commercialization. The Development/R\&D phase is set in motion by initial conceptualizations of a service (the solution to a problem) and business model. Because, in this phase, the core activities are service or product definitions, investment in new technologies and collaboration with technology providers, R\&D (basic and applied research) and technology play a dominant role. The business model enters the second phase when the service is launched on the market. Activities in this phase include testing service concepts in focus groups, conducting field experiments, rolling out the technology, testing alpha and beta versions of the service, and carrying out (small-scale) roll-out on the market. The business model enters the third phase once the service reaches critical mass, after market experiments have proven successful. Core activities in this phase are retaining rather than capturing market share, managing the commercial exploitation on a day-to-day basis, and focusing on operations and maintenance. As such, the Commercialization phase subsumes the previously identified stages of market offering, maturity and decline. In reality, the life cycle of business models may not always follow the linear path outlined here. There may be iterations, in particular when things do not go as planned.

\section{Research model}

Because there are very few studies on business model dynamics, we cannot rely on existing models or hypotheses. We expect that the impact of external drivers on internal business model components will be different in each phase. In the Development/R\&D phase, we expect technology to be the main driver behind new business model development. Specifically, the emergence of new mobile, wireless and data networks, like the Internet, allow for an increased reach of businesses, while middleware, web services and multimedia applications at the same time offer new opportunities for enriched, customized and secure communication. However, new business models can also be driven by market developments, for instance changing customer demand or new entrants on the market, or by regulatory changes, for example liberalization.

In the Implementation/Roll-out phase, regulators and competitors become aware of the new service, and they will examine possible regulatory implications and prepare a strategic response, for instance by demanding stricter regulation. As a result, it has to be certain that the new service complies with regulation regarding issues like fair competition, privacy, intellectual property rights and content restrictions. Changes in market factors, for instance competitors copying the service concept, and technology, i.e. the availability of more innovative or cheaper solutions, can affect the service and business model, but we expect them to be less conditional in nature than regulation, and assume their impact on the business model to be less pronounced in this phase.

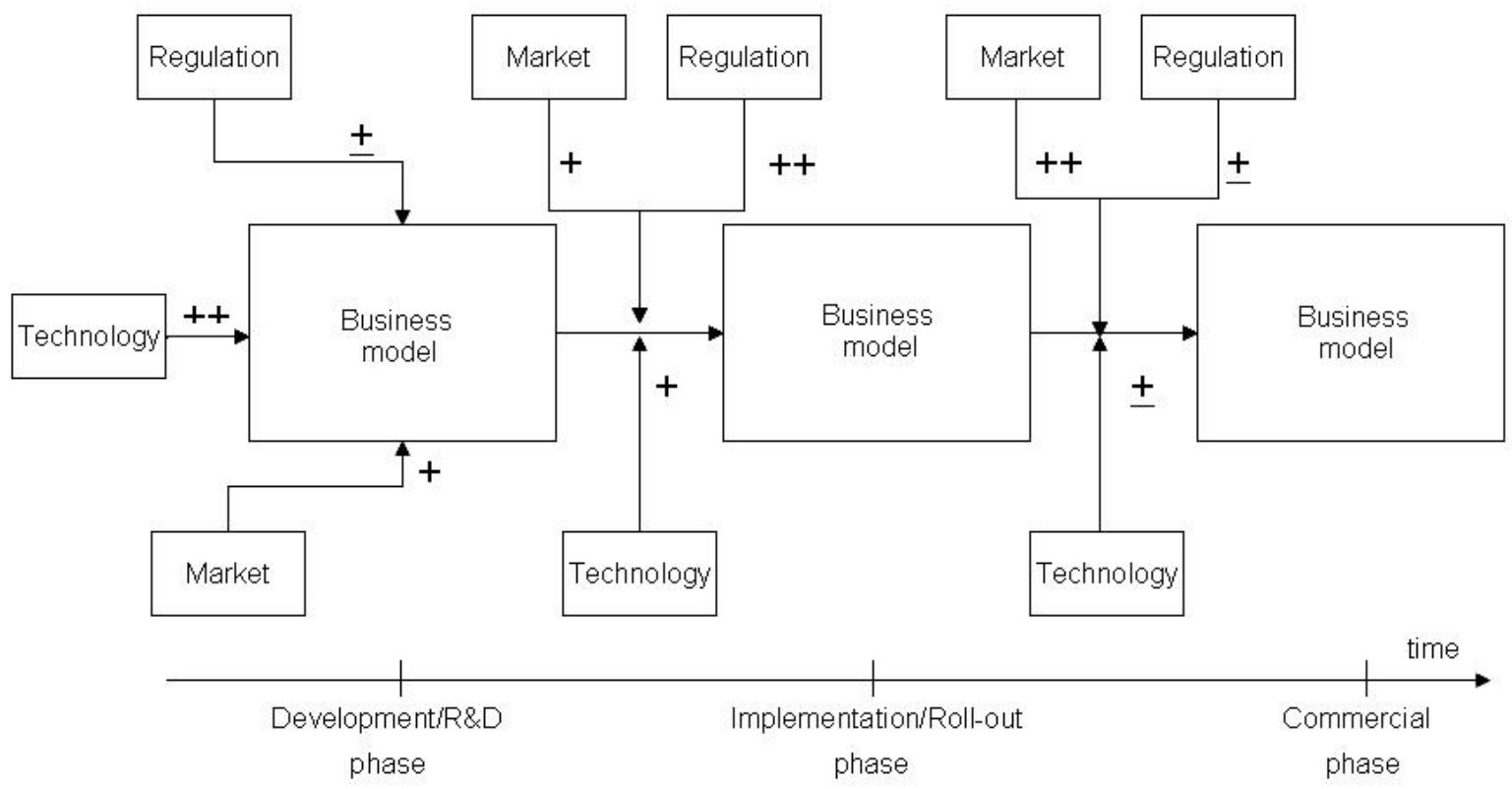

Figure 1: Dynamic business model framework: Propositions 
Finally, in the Commercial phase, we expect market-related drivers to play an important role, with the focus being on retaining customers and with competitors starting to revise their business models in response to the service offering. Regulation is aimed more at monitoring existing rules to ensure they are observed, and technological drivers have a minor impact, since changes in the business model will mainly involve scalability, operations, and maintenance, requiring periodical updates rather than a full revision of the technological architecture.

In summary, we outline the following hypotheses, see Figure 1:

- $\quad H 1$ : Technological drivers are most relevant $(++)$ in the Development/R\&D phase, decreasing to medium (+) in the Implementation/Roll-out and low $( \pm)$ in the Commercial phase.

- $\quad \mathrm{H} 2$ : Market-related drivers are most relevant $(++)$ in the Commercial phase and less (+) in the other two phases.

- H3: Regulatory drivers are most important $(++)$ in the Implementation/Roll-out phase, and almost irrelevant $( \pm)$ in the other phases.

As mentioned earlier, we do not examine the way the different components (service/product, technological architecture, organizational or financial arrangements) of a business model are affected, but instead focus on the impact of external drivers on the business model as a whole.

\section{Method}

As Yin and Heald [23] argue, case surveys are particularly suited when there is a heterogeneous collection of case studies exists and researchers are interested in their characteristics rather than the conclusions of the authors. It is an approach that combines advantages of survey research, i.e. dealing with a large number of observations based on individual in depth, and qualitative cases. Case surveys allow for quantitative analyses and statistical generalizations, while capitalizing on the richness of case material [10]. We used content analysis as a tool to analyze the data from the case survey.

\subsection{Case selection}

We selected over sixty case descriptions involving the business models from companies like Abcam.com, Blockbuster, Centagenetix, Disney, NTT DoCoMo, Electronic Arts, FedEx, Google, Intel, Matsui, MySQL, Non-stop Yacht, Paypal, Cisco, Webraska and Yahoo!. To ensure cross-case comparability, we used business school teaching cases, because their structure is more or less the same. In addition, they provide the longitudinal descriptions needed to test the time dimension of our hypotheses, and they are readily available. The descriptions involved cases that were developed between 1999 and 2004, a period when business cases involving mobile and Internet technology triggered discussions on business models. The cases were widely available in business school repositories, for instance Harvard, the University of Virginia, Stanford, Ivey and the European Cases Clearing House. Not all cases dealt with all three phases of our research model, simply because the services involved had not yet reached mass market or because they involved established companies that were already in the last phase of their life cycle. Other cases showed multiple feedback loops between the various phases. To deal with the heterogeneity we encountered, we decided to look at each phase of a case in isolation, which resulted in 97 units of analysis.

Table 1: Case characteristics $(\mathrm{N}=97)$

\begin{tabular}{|l|l|l|}
\hline Variable & Category & Number of cases \\
\hline Industry sector & (Mobile) telecommunications & 14 \\
\cline { 2 - 3 } & Software & 7 \\
\cline { 2 - 3 } & Healthcare / High-tech & 8 \\
\cline { 2 - 3 } & Consumer goods & 13 \\
\cline { 2 - 3 } & Finance & 8 \\
\cline { 2 - 3 } & Entertainment & 11 \\
\cline { 2 - 3 } & Intermediary services & 26 \\
\cline { 2 - 3 } & Logistics / Transport & 10 \\
\hline Nodal company age & Start-up & 59 \\
\cline { 2 - 3 } & Established & 38 \\
\hline Nodal company size & Small (<150 employees) & 57 \\
\cline { 2 - 3 } & Large & 40 \\
\hline Phase & Development/R\&D & 36 \\
\cline { 2 - 3 } & Implementation/Roll-out & 38 \\
\cline { 2 - 3 } & Commercial & 23 \\
\hline
\end{tabular}


We selected cases from various industries to enhance the applicability of our results (see Table 1 for case characteristics). Our selection contained about as many start-ups as it did established companies, and the same applied to small and large companies. All phases contain a reasonable proportion of cases, although phase III is somewhat underrepresented.

\subsection{Coding protocol}

We developed a coding protocol specifying the variables to be coded (available upon request from the first author). We included background variables (company size, age, strategy, culture, technology fit, industry sector, innovation type) and driver variables in the protocol. As far as the background variables are concerned, we used categorical scales to specify industry sector, nodal company size and nodal company age. We divided the technological drivers into general technological trends: digitization, processing power, miniaturization, mobile technology, technical integration, positioning technology, intelligent systems, interoperability security, and natural interfaces [4]. To this list we added Internet technology, standardization bodies, incremental nature of technology and degree of technological sophistication. As far as market-related drivers are concerned, we included Porter's forces of entry barriers, threat of substitution, suppliers' bargaining power, firms' rivalry [17], competitors' business models, vertical and horizontal integration, and financial and general innovation climate. With regard to market demand, we used the income levels of customers, potential target groups, degree of customer power, Internet adoption, mobile adoption, as well as a set of socio-economic trends, i.e. individualization, self-chosen collectivity, informalization, intensivitization, feminization, ageing of population and increasing cultural diversity [9]. The regulatory drivers we identified are deregulation, regulation from national regulatory authority, economic regulation, legal regulation, security regulation and customer protection regulation. For each of the driver variables, we specified objective criteria to code the significance of the drivers on an ordinal scale (a strong, weak, questionable or absent influence). In addition to the pre-coded driver variables, we added free-format fields in the protocol, to allow the coders to add other relevant drivers they might encounter in the case descriptions.

We developed a detailed manual, as is common in content analysis, on how to use the protocol, outlining each individual step. The manual explained how the coders should distinguish the three phases. The start of the Development/R\&D phase was defined simply as the moment when the initially idea about a service concept or technology was conceived. The start of the Implementation/Roll-out phase was defined as the moment the service was launched on the market. To indicate the start of the Commercial phase, indicators were specified in the following order of importance: reaching critical mass, shift of focus from market expansion toward customer retention, the launch of version 1.0 of the service, and the targeting new markets. In addition to the phasing, the protocol defined each variable in the protocol, or a reference to literature. Only information that was found in the case description could be coded. In case of uncertainty about the meaning of variables or values, the coders were instructed to contact the principal authors.

\subsection{Coding the cases}

Because using several coders is essential in ensuring reliable case survey research [10], [23], we used four coders to analyze the cases. Each case was assigned at random to two of the four coders. The first step in coding the cases involved reading the material and deciding on the start and end dates of the phases for the individual cases. The coders compared and discussed the phases, to make sure that both coders would use the same material in relation to the specific phasing. As we expected, feedback loops, i.e. multiple iterations within a specific phase, are common, and shifts from the second towards the third phase did lead to discussions about the proper phasing. Finally, the coders coded the variables of the protocol individually. After all the cases had been coded, we recoded the freeformat driver variables into ordinal variables wherever possible.

\subsection{Reliability}

Because we relied on observer interpretations, we had to compute intercoder reliability measures. As far as external drivers are concerned, the data indicated that coders usually agreed on whether a driver had been of any importance, but typically disagreed on the level of influence. We therefore recoded the external drivers to binary values, i.e. 'influence' and 'no influence'. After this transformation, we found that the coders agreed in more than $70 \%$ of the cases for 62 out of 65 driver variables $(94 \%)$ and for all our background variables. To correct for any agreement that may be attributed to chance, we also computed Cohen's Kappa. However, we found that variation of the variables was typically low (many drivers were predominantly coded 'no influence'), resulting in a disproportionately low Kappa values. For some variables, for example, there was agreement in over $90 \%$ of the cases, while Kappa values were lower than 0.6. We therefore believe that, for this type of data, the level of agreement can be used to measure intercoder reliability. We removed three variables where the level of agreement was lower than $70 \%$. After establishing the reliability of the case coding, we had to choose one of the two available data sets. Because taking averages of two data-sets is impossible, we decided to use the data-set of the coders that scored high on the Kappa values. 


\section{Results}

We grouped all the drivers to aggregate measures for each of the three categories (i.e. technology, market and regulation). We calculated two types of aggregate measures: a ratio-scale variable adding the total number of lowerlevel drivers with the value 'influence' for the respective categories, and a binary variable coded ' 1 ' for cases with one or more of the low-level drivers coded 'influence' and ' 0 ' when none of the drivers in question had been important to the case.

\subsection{Technological drivers}

Our first hypothesis is that technological drivers are most important in the first phase, with their importance decreasing to medium in the second and low in the third phase. We tested this by logistic regression analysis, using the binary aggregate technology driver variable as a dependent variable, and the phase variable as a predictor. The phase variable is recoded into two dummy variables, with base value referring to phase I, as we want to see if the importance of the driver is lower in the other phases compared to phase I. Table 2 contains the logistic regression model and shows that, compared to phase I, the dummy variables provide significant prediction to the likely hood of technological drivers to occur. The model as a whole has explained variance of .16 (Cox \& Snell) or .22 (Nagelkerke). We also executed linear regression analysis, taking the ratio-scale aggregate technology driver variable as a dependent. Table 3 indicates that standardized coefficients are significant for phase II and III. The difference in explained variance between Table 2 and 3 is explained by the different type of regression analysis: i.e., logistic versus linear. The results confirm our hypothesis that technological drivers are less important in later phases than they are in phase I (see Table 2 and 3).

Table 2: Logistic regression for binary technology driver

\begin{tabular}{|l|l|l|l|l|}
\hline & & \multicolumn{3}{|c|}{$95 \% \mathrm{Cl}$ for $\operatorname{exp~b}$} \\
\hline & $\mathrm{B}(\mathrm{SE})$ & Lower & $\operatorname{exp~b}$ & Upper \\
\hline Constant & $0.22(.34)$ & & 1.25 & \\
\hline Phase II (dummy) & $-1.39^{* *}(.51)$ & 0.09 & 0.25 & 0.67 \\
\hline Phase III (dummy) & $-2.58^{\star *}(.81)$ & 0.02 & 0.08 & 0.38 \\
\hline
\end{tabular}

Note: Hosmer \& Lemeshow p-value $=1, \mathrm{R}^{2}=.16$ (Cox \& Snell), .22 (Nagelkerke). ${ }^{*} p<.05 ;{ }^{* *} p<0.01 ;{ }^{* * *} p<0.001$

Table 3: Linear regression for ratio-scale technology driver

\begin{tabular}{|l|l|l|l|}
\hline & B & SE B & $\beta$ \\
\hline Constant & 1.22 & 0.18 & \\
\hline Phase II (dummy) & -0.80 & 0.26 & $-.37^{* *}$ \\
\hline Phase III (dummy) & -1.01 & 0.29 & $-.37^{* * *}$ \\
\hline
\end{tabular}

Note: $F=7.48, d f=94,2, p<0.001 . R^{2}=.14 .{ }^{*} p<.05 ;{ }^{* *} p<0.01 ;{ }^{* *} p<0.001$.

We checked whether the results would be different depending on business size and business age. As far as the logistic regression analyses for small businesses is concerned, the coefficient for phase II is significant, while the coefficient for phase III is not (see Table 4). This may be explained by the fact that there were no technological drivers present in any of the 16 cases of small businesses in phase III.

Table 4: Logistic regression for binary technology driver, small businesses

\begin{tabular}{|l|l|l|l|l|}
\hline & & \multicolumn{3}{|c|}{$95 \% \mathrm{Cl}$ for $\operatorname{exp~b}$} \\
\hline & $\mathrm{B}(\mathrm{SE})$ & Lower & $\operatorname{exp~b}$ & Upper \\
\hline Constant & $0.29(.44)$ & & 1.33 & \\
\hline Phase II (dummy) & $-2.13^{* *}(.76)$ & 0.03 & 0.12 & 0.53 \\
\hline Phase III (dummy) & -21.49 & 0.00 & 0.00 & 0.00 \\
\hline
\end{tabular}

Note: Hosmer \& Lemeshow p-value $=1, R^{2}=.29$ (Cox \& Snell), .42 (Nagelkerke). ${ }^{*} p<.05 ;{ }^{* *} p<0.01 ;{ }^{* * *} p<0.001$.

With regard to the large businesses, the model has much lower explained variance, and none of the coefficients are significant. Having said that, there is no significant relationship between business size and the number of drivers found regardless of the phase $(x 2(1)=2.02$, ns), which means it is not possible to conclude that technological drivers are not important at all for these type of companies. 
Table 5: Logistic regression for binary technology driver, large businesses

\begin{tabular}{|l|l|l|l|l|}
\hline & & \multicolumn{3}{|c|}{$95 \% \mathrm{Cl}$ for $\operatorname{exp~b}$} \\
\hline & $\mathrm{B}(\mathrm{SE})$ & Lower & $\operatorname{exp~b}$ & Upper \\
\hline Constant & $0.13(.52)$ & & 1.14 & \\
\hline Phase II (dummy) & $-0.64(.73)$ & 0.13 & 0.53 & 2.20 \\
\hline Phase III (dummy) & $-1.39(.95)$ & 0.04 & 0.25 & 1.62 \\
\hline
\end{tabular}

Note: Hosmer \& Lemeshow p-value $=1, \mathrm{R}^{2}=.06$ (Cox \& Snell), .08 (Nagelkerke). ${ }^{*} p<.05 ;{ }^{* *} p<0.01 ;{ }^{* *} p<0.001$

In the case of start-up companies, we see a phenomenon similar to the one found with small companies. Again, comparing phase I with phase II, the same conclusions are drawn, but because there were no start-ups in the datasets for phase III, any claims are bound to be questionable. Also, as was the case for the large businesses, the hypotheses cannot be accepted for established businesses. We did find that there is a relationship between business age and technological drivers: $\mathrm{X} 2(1)=4.69, \mathrm{p}<0.05$.

Table 6: Logistic regression for binary technology driver, startups

\begin{tabular}{|l|l|l|l|l|}
\hline & & \multicolumn{3}{|c|}{$95 \% \mathrm{Cl}$ for $\operatorname{exp~b}$} \\
\hline & $\mathrm{B}(\mathrm{SE})$ & Lower & $\operatorname{exp~b}$ & Upper \\
\hline Constant & $0.10(.44)$ & & 1.10 & \\
\hline Phase II (dummy) & $-1.99^{* \star}(.76)$ & 0.14 & 0.03 & 0.60 \\
\hline Phase III (dummy) & -21.30 & 0.00 & 0.00 & 0.00 \\
\hline
\end{tabular}

Note: Hosmer \& Lemeshow p-value $=1, \mathrm{R}^{2}=.26$ (Cox \& Snell), .39 (Nagelkerke). ${ }^{*} p<.05 ;{ }^{* *} p<0.01 ;{ }^{* * *} p<0.001$

Table 7: Logistic regression for binary technology driver, established companies

\begin{tabular}{|l|l|l|l|l|}
\hline & & \multicolumn{3}{|c|}{$95 \% \mathrm{Cl}$ for $\operatorname{exp~b}$} \\
\hline & $\mathrm{B}(\mathrm{SE})$ & Lower & $\operatorname{exp~b}$ & Upper \\
\hline Constant & $0.41(.53)$ & & 1.50 & \\
\hline Phase II (dummy) & $-0.81(.74)$ & 0.10 & 0.44 & 1.92 \\
\hline Phase III (dummy) & $-1.50(.97)$ & 0.03 & 0.22 & 1.49 \\
\hline
\end{tabular}

Note: Hosmer \& Lemeshow p-value $=1, \mathrm{R}^{2}=.07$ (Cox \& Snell), .10 (Nagelkerke). ${ }^{*} p<.05 ;{ }^{* *} p<0.01 ;{ }^{* * *} p<0.001$

We find similar results (not displayed here) in linear regression analysis with the ratio-scale technology driver measure: the model has a better fit for cases involving small start-up companies, but no longer for large established companies.

To summarize, we find that the cases support our hypothesis that technological drivers are more important in the Development/R\&D phase than in the other phases, although it would appear that this only applies small start-up cases.

\subsection{Market-related drivers}

Because we hypothesized that market-related drivers are most important in phase III, and less so in phase II and I, we constructed two dummy predictor variables for the phase variable, with base value phase III. The results (Table 8 ) indicate that there is no significant difference with regard to the importance of market-related drivers when comparing phases II and I to phase III.

Table 8: Logistic regression for binary market-related driver (base value = Phase III)

\begin{tabular}{|l|l|l|l|l|}
\hline & & \multicolumn{3}{|c|}{$95 \% \mathrm{Cl}$ for $\operatorname{exp~b}$} \\
\hline & $\mathrm{B}(\mathrm{SE})$ & Lower & $\operatorname{exp~b}$ & Upper \\
\hline Constant & $0.26(.42)$ & & 1.30 & \\
\hline Phase I (dummy) & $0.99(.58)$ & 0.86 & 2.70 & 8.41 \\
\hline Phase II (dummy) & $-0.58(.53)$ & 0.20 & 0.56 & 1.59 \\
\hline
\end{tabular}

Note: Hosmer \& Lemeshow p-value $=1, \mathrm{R}^{2}=.10$ (Cox \& Snell), .13 (Nagelkerke). ${ }^{*} p<.05 ;{ }^{* *} p<0.01 ;{ }^{* * *} p<0.001$

When we take phase I as a reference (i.e. use dummy variables like in the Technology driver model), however, we find that there are significant differences in importance when comparing phase I to phase III, and there is an indication that there is a difference (although not a significant one) between phases I and II. So, the data suggests that our hypothesis should be rejected, i.e. market-related drivers are most prominent in phase I. The same 
alternative hypothesis is supported by the linear regression analysis of the ratio-measure for the market driver (see Table 9).

Table 9: Logistic regression for binary market-related driver (base value $=$ Phase I)

\begin{tabular}{|l|l|l|l|l|}
\hline & & \multicolumn{3}{|c|}{$95 \% \mathrm{Cl}$ for $\operatorname{exp~b}$} \\
\hline & $\mathrm{B}(\mathrm{SE})$ & Lower & $\operatorname{exp~b}$ & Upper \\
\hline Constant & $1.25^{\star *}(.40)$ & & 3.50 & \\
\hline Phase II (dummy) & $-1.57(.52)$ & 0.08 & 0.21 & 0.57 \\
\hline Phase III (dummy) & $-0.99^{\star *}(.58)$ & 0.12 & 0.37 & 1.16 \\
\hline
\end{tabular}

Note: Hosmer \& Lemeshow p-value $=1, \mathrm{R}^{2}=.10$ (Cox \& Snell), .13 (Nagelkerke). ${ }^{*} p<.05 ;{ }^{* *} p<0.01 ;{ }^{* * *} p<0.001$

Table 10: Linear regression for ratio-scale market-related driver (base value $=$ Phase I)

\begin{tabular}{|l|l|l|l|}
\hline & B & SE B & $\beta$ \\
\hline Constant & 0.65 & 0.26 & \\
\hline Phase II (dummy) & 1.01 & 0.34 & $.37^{* *}$ \\
\hline Phase III (dummy) & 0.09 & 0.33 & .80 \\
\hline
\end{tabular}

Note: $F=6.57, d f=94,2, p \leq 0.01 R^{2}=.12 .{ }^{*} p<.05 ;{ }^{* *} p<0.01 ;{ }^{* * *} p<0.001$.

When we divide the output based on company size and age variables, we again find there are differences in model fit between the categories. We find remarkably higher explained variance, and more significant coefficients, for small businesses and start-ups (see Tables 11 and 12).

Table 11: Logistic regression for binary market-related driver, small businesses

\begin{tabular}{|l|l|l|l|l|}
\hline & & \multicolumn{3}{|c|}{$95 \%$ Cl for $\operatorname{exp~b}$} \\
\hline & $\mathrm{B}(\mathrm{SE})$ & Lower & $\operatorname{exp~b}$ & Upper \\
\hline Constant & $1.79^{\star *}(.62)$ & & 6.00 & \\
\hline Phase II (dummy) & $-2.35^{*}(.77)$ & 0.02 & 0.10 & 0.43 \\
\hline Phase III (dummy) & $-1.79^{* *}(.82)$ & 0.03 & 0.17 & 0.83 \\
\hline
\end{tabular}

Note: Hosmer \& Lemeshow p-value $=1, \mathrm{R}^{2}=.20$ (Cox \& Snell), .26 (Nagelkerke). ${ }^{*} p<.05 ;{ }^{* *} p<0.01 ;{ }^{* * *} p<0.001$.

Table 12: Logistic regression for binary market-related driver, start-ups

\begin{tabular}{|l|l|l|l|l|}
\hline & & \multicolumn{4}{|c|}{$95 \%$ Cl forp b } \\
\hline & $\mathrm{B}(\mathrm{SE})$ & Lower & $\operatorname{exp~b}$ & Upper \\
\hline Constant & $1.45^{\star *}(.56)$ & & 4.25 & \\
\hline Phase II (dummy) & $-1.89^{* *}(.70)$ & 0.04 & 0.15 & 0.60 \\
\hline Phase III (dummy) & $-1.58^{*}(.76)$ & 0.05 & 0.21 & 0.91 \\
\hline
\end{tabular}

Note: Hosmer \& Lemeshow p-value $=1, \mathrm{R}^{2}=.14$ (Cox \& Snell), .19 (Nagelkerke). ${ }^{*} p<.05 ;{ }^{* *} p<0.01 ;{ }^{* * *} p<0.001$

With regard to the large and established businesses, the model does not fit (see Tables 13 and 14). However, we find no significant relation between the drivers and business size $(x 2(1)=0.04, n s)$ nor business age $(x 2(1)=0.50$, $\mathrm{ns})$.

Table 13: Logistic regression for binary market-related driver, large businesses

\begin{tabular}{|l|l|l|l|l|}
\hline & & \multicolumn{3}{|l|}{$95 \% \mathrm{Cl}$ forp b } \\
\hline & $\mathrm{B}(\mathrm{SE})$ & Lower & $\operatorname{exp~b}$ & Upper \\
\hline Constant & $0.69(.55)$ & & 2.00 & \\
\hline Phase II (dummy) & $-0.69(.74)$ & 0.12 & 0.50 & 2.14 \\
\hline Phase III (dummy) & $0.000(.89)$ & 0.17 & 1.00 & 5.77 \\
\hline
\end{tabular}

Note: Hosmer \& Lemeshow $p$-value $=1, \mathrm{R}^{2}=.027$ (Cox \& Snell), .037 (Nagelkerke). ${ }^{*} p<.05 ;{ }^{* *} p<0.01 ;{ }^{* * *} p<0.001$.

When we tested the linear regression model based on the ratio-scale measure for aggregate market-related drivers, the results are similar: explained variance and significance of coefficients increases for start-ups and small companies, while the model no longer fits for established, large companies. 
Table 14: Logistic regression for binary market-related driver, established businesses

\begin{tabular}{|l|l|l|l|l|}
\hline & & \multicolumn{4}{|l|}{$95 \% \mathrm{Cl}$ for $\operatorname{exp~b}$} \\
\hline & $\mathrm{B}(\mathrm{SE})$ & Lower & $\operatorname{exp~b}$ & Upper \\
\hline Constant & $1.01(.584)$ & & 2.75 & \\
\hline Phase II (dummy) & $-1.15(.780)$ & 0.07 & 0.32 & 1.47 \\
\hline Phase III (dummy) & $0.09(1.00)$ & 0.15 & 1.09 & 7.8 \\
\hline
\end{tabular}

Note: Hosmer \& Lemeshow p-value $=1, R^{2}=.073$ (Cox \& Snell), .10 (Nagelkerke). ${ }^{*} p<.05 ;{ }^{* *} p<0.01 ;{ }^{* * *} p<0.001$.

To summarize, we have to reject our initial hypothesis that market-related drivers are most important in the third phase, and instead argue that they are most relevant in the first phase. Again, this hypothesis applies only to small start-ups.

\subsection{Regulatory drivers}

With regard to regulatory drivers, we hypothesized that they are most important in the second phase, and less so in the first and third phase. However, neither binary logistic regression nor linear regression indicates any significant differences when comparing phases.

Table 15: Logistic regression for binary regulatory driver

\begin{tabular}{|l|l|l|l|l|}
\hline & & \multicolumn{4}{|c|}{$95 \% \mathrm{Cl}$ for $\operatorname{exp~b}$} \\
\hline & $\mathrm{B}(\mathrm{SE})$ & Lower & $\operatorname{exp~b}$ & Upper \\
\hline Constant & $-1.89^{\star * *}(.48)$ & & 0.15 & \\
\hline Phase I (dummy) & $0.63(.63)$ & 0.55 & 1.89 & 6.42 \\
\hline Phase III (dummy) & $0.61(.70)$ & 0.47 & 1.83 & 7.19 \\
\hline
\end{tabular}

Note: Hosmer \& Lemeshow p-value $=1 . \mathrm{R}^{2}=.01$ (Cox \& Snell), .02 (Nagelkerke). ${ }^{*} p<.05 ;{ }^{* *} p<0.01 ;{ }^{* * *} p<0.001$

Table 16: Linear regression for ratio-scale regulatory driver

\begin{tabular}{|l|l|l|l|}
\hline & $\mathrm{B}$ & SE B & $\beta$ \\
\hline Constant & 0.13 & 0.09 & \\
\hline Phase I (dummy) & 0.20 & 0.13 & .18 \\
\hline Phase III (dummy) & 0.17 & 0.15 & .13 \\
\hline
\end{tabular}

Note: $F=1.37, \mathrm{df}=94,2$, ns. $\mathrm{R}^{2}=.03 .{ }^{*} p<.05 ;{ }^{* *} p<0.01 ;{ }^{* * *} p<0.001$.

Differentiating between business size and age does not yield better models, with Nagelkerke R Square still lower than 0.05. Cross tabulations also do not indicate significant relationships between the binary regulatory driver measure on the one hand, and business size $(x 2(1)=3.60, n s)$ or business age $(x 2(1)=4.46$, ns). This means that we have to reject our hypothesis that regulatory drivers are most relevant in the second phase. Instead, we propose that these drivers play a minor role throughout all phases, regardless of company size and age.

\section{Limitations}

As with any case survey research, our findings are constrained by the quality of the original case descriptions [23]. The case material was originally collected for other purposes, and may have focused on specific fields of interest or educational purposes. However, we did find, for example, that technological drivers were mentioned, contrary to what one might expect from business scholars. Business cases that are used for educational purposes may be oversimplified, ignoring the complexity of the business models involved. However, a positive effect of this oversimplification may be that the relevant issues are made more explicit. Another limitation of using existing cases is that the cases are related to different markets and industry sectors. However, most of the cases covering the period 1999-2004 focus on the impact of information and communication technology, more specifically Internet and mobile technology, and in that sense they have a great deal in common.

Because data collection always involved the risk of interpretation and bias, using existing cases from different authors reduces risk of personal bias. The reason we decided to use existing cases was to allow for statistical generalization across a large number of cases. Future case survey research may be improved by combining several types of case descriptions, i.e. both teaching cases and research cases, or by validating the coding results with company stakeholders [10].

It was sometimes difficult to assign the right phasing to the cases, and in particular the transition from the second to the third phase can be troublesome. In some cases we found contradictory indicators in the case descriptions, and 
discussion was needed among the coders and researchers to come to an agreement. This underlines the importance of ensuring a strict operationalization of phasing models for similar research in the future. Another issue with regard to phasing has to do with the fact that we did not have enough cases that cover all three phases. When we started the case survey we assumed, incorrectly as it turned out, that we would have enough material covering all three phases in individual case descriptions, and that we could analyze the effects of the various external drivers on the sequential phases across cases. Our analysis was limited by the fact that most cases involved only one of the phases, and in some cases two.

Although we instructed our coders to limit themselves to the information contained in the cases, we often found that common sense would dictate that certain drivers are actually important, even though they were not mentioned explicitly in the case descriptions. In retrospect, we might have given the coders more freedom to use their own interpretation, although that would have inevitably created bias towards more familiar cases.

\section{Conclusions and discussion}

The goal of this paper was to identify the external drivers that are most relevant in the various phases of a business model life cycle. Our study indicates that technological and market-related drivers are most relevant in the stages of service conceptualization and the underlying technological development. This was what we expected to find for technological drivers, but for market-related drivers it is contrary to our expectations. Apparently, decisions about new services and underlying business models are fueled more by market developments than by later adjustments to choices that were made at an earlier stage. Existing studies on dynamic business models are biased towards the music industry, and usually examine individual cases. In this study, we included cases from various sectors, which means that, although the number of cases we studied may be limited, our results have a broader external validity.

We found very few cases where regulatory drivers play a role (only $18 \%$ of all cases) and, contrary to what we expected in advance, we found no relationship between the phase in the life cycle and the importance of this type of drivers. This is remarkable, because anecdotic evidence tells us that issues like IPR (e.g., Napster, YouTube) and competition engineering (e.g., DoCoMo's I-mode) do play a role. Apparently, these cases are exceptions rather than the rule. Instead, we propose that regulation plays only a minor role in all the phases of a business model life cycle.

Our model, which describes the impact of external drivers on business models, is much more applicable for the business models of small, start-up companies than it is for large, established businesses. Although external drivers are also important in the development of new business models of larger, established companies, their importance does not appear to change over time.

Combining our findings leads to the adjusted model in Figure 2. Our findings are important from an academic point of view, because they help us understand what drives business model dynamics. From a practical point of view, our findings imply that, when developing new services and products and their underlying business models, companies need to be aware of the role technology and market forces play in the first phase of business model development. Furthermore, design methodologies for business models that take into account their dynamics need to differentiate between start-ups and established firms.

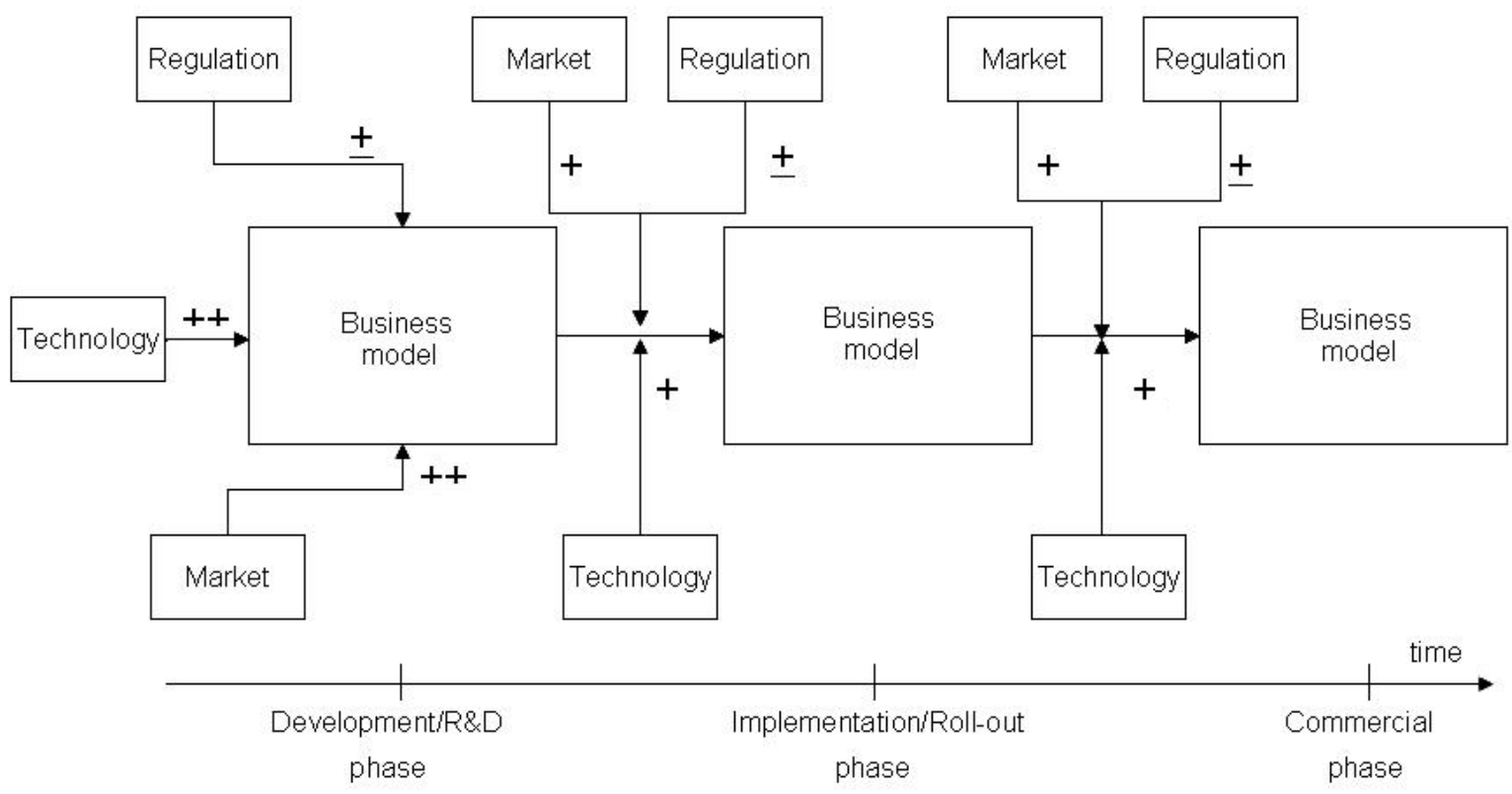

Figure 2: Adjusted dynamic business model framework for small startups 


\section{References}

[1] A. Afuah and C. Tucci, Internet Business Models and Strategies. Boston McGraw-Hill, 2003.

[2] P. Andries, K. Debackere, and B. Van Looy, Effective business model adaptation strategies for new technologybased ventures, in Proceedings 9th PREBEM Conference on Business Economics, Management and Organization Science, Amersfoort, The Netherlands, 2006.

[3] H. Bouwman, H. de Vos, \& T. Haaker, Mobile Service inovation and Business Models, Heidelberg: Springer, 2008.

[4] H. Bouwman, B. Van den Hooff, L. v. d. Wijngaert, and J. v. Dijk, Information \& Communication Technology in Organizations. London: Sage Publications Inc, 2005.

[5] R. A. Burgelman, A Process Model of Internal Corporate Venturing in the Diversified Major Firm, Administrative Science Quarterly, vol. 28, no. 2, pp. 223-244, 1983.

[6] H. Chesbrough and R. S. Rosenbloom, The role of the business model in capturing value from innovation: evidence from Xerox Corporation's technology spin-off companies, Industrial and Corporate Change, vol. 11, no. 3, pp. 529-555, 2002.

[7] T. Haaker, E. Faber, and H. Bouwman, Balancing customer and network value in business models for mobile services, International Journal of Mobile Communications, vol. 4, no. 6, pp. 645-661, 2006.

[8] J. Hedman and T. Kalling, The business model concept: theoretical underpinnings and empirical illustrations, European Journal of Information Sciences, vol. 12, no. 1, pp. 49-59, 2003.

[9] P. Idenburg, Oog voor de toekomst: Over marketing en consumenten in een veranderende samenleving. Haarlem: Scriptum, 2004.

[10] R. Larsson, Case survey methodology: Quantitative analysis of patterns across case studies, Academy of Management Review, vol. 39, no. 6, pp. 1515-1546, 1993.

[11] I. Maclnnes, Dynamic business model framework for emerging technologies, International Journal of Services Technology and Management, vol. 6, no. 1, pp. 3-19, 2005.

[12] H. Mason and T. Rohner, The Venture Imperative, A New Model for Corporate Innovation. Boston: Harvard Business Press, 2002.

[13] M. Morris, M. Schindehutte, and J. Allen, The entrepeneur's business model: toward a unified perspective, Journal of Business Research, vol. 58, no. 6, pp. 726-735, 2005.

[14] A. Osterwalder and Y. Pigneur, An e-Business Model Ontology for Modeling e-Business, in Proceedings 15th Bled Electronic Commerce Conference, Bled, Slovenia, 2002.

[15] A. G. Pateli and G. M. Giaglis, A research framework for analyzing eBusiness models, European Journal of Information Sciences, vol. 13, no. 4, pp. 302-314, 2004.

[16] A. G. Pateli and G. M. Giaglis, Technology innovation-induced business model change: a contingency approach, Journal of Organizational Change Management, vol. 18, no. 2, pp. 167-183, 2005.

[17] M. E. Porter, Competitive advantage; creating and sustaining superior performance. New York: Free Press, 1985.

[18] E. M. Rogers, Diffusion of Innovations. New York: The Free Press, 1962.

[19] P. B. Seddon and G. P. Lewis, Strategy and Business Models: What's the Difference?, in Proceedings 7th Pacific Asia Conference on Information Systems, Adelaide, South Australia, 2003.

[20] S. M. Shafer, H. J. Smith, and J. C. Linder, The power of business models, Business Horizons, vol. 48, no. 3, pp. 199-207, 2005.

[21] J. Tidd, J. Bessant, and K. Pavitt, Managing Innovation. New York: Wiley, 2001.

[22]V. L. Vaccaro and D. Y. Cohn, The Evolution of Business Models and Marketing Strategies in the Music Industry, JMM - The International Journal on Media Management, vol. 6, no. 1-2, pp. 46-58, 2004.

[23] R. K. Yin and K. A. Heald, Using the Case Survey Method to Analyze Policy Studies, Administrative Science Quarterly, vol. 20, no. 3, pp. 371-381, 1975. 